\title{
Factores asociados al consumo de cocaína en usuarios de heroína inyectable en Colombia
}

\author{
Dedsy Yajaira Berbesi-Fernández, ${ }^{1}$ Angela Segura-Cardona, ${ }^{1}$ Liliana Patricia Montoya-Velez, ${ }^{1}$ \\ Andres Felipe Ramirez Medina'
}

Artículo original

\section{ABSTRACT}

\section{Introduction}

People who use heroin and cocaine have more sexual activity, risk behaviors associated with injection and inconsistent condom use, increasing the risk of sexually transmitted infections and the presence of HIV.

\section{Objective}

To identify socio-demographic factors, sexual behavior and injection heroin risk in users, according to additional consumption of cocaine in five cities in Colombia.

\section{Method}

1017 injecting drug users over 18 years were surveyed; by sampling generated by the respondent, reasons of raw prevalence (OR) were calculated and adjusted through logistic regression, with their respective according to cocaine consumption.

\section{Results}

$60 \%$ of injected heroin users also used cocaine; this population were largely male, unmarried, under 25 years, belonging to low socioeconomic status and involved in dealing drugs. Risk behaviors in the last injection of the drug were higher in those who also used cocaine. Condom use at last intercourse was lower and a higher prevalence of HIV was observed in cocaine users.

\section{Discussion and conclusion}

It was determined that injecting drug users who use cocaine are faced with a high probability of HIV and less condom use, as well as greater sexual risk behavior and related to injection. Attention is required with preventive and social measures to reduce the use of psychoactive drugs, polydrug use and the consequences of these drugs in Colombia.

Key words: Heroin, cocaine, sexual behavior, HIV, hepatitis C.

\section{RESUMEN}

\section{Introducción}

Las personas que consumen heroína y cocaína presentan una mayor actividad sexual, conductas de riesgo relacionadas con la inyección y un uso inconsistente del condón, lo que aumentaría el riesgo de infecciones de transmisión sexual y la presencia de $\mathrm{VIH}$.

\section{Objetivo}

Identificar factores sociodemográficos, conductas sexuales y de inyección en usuarios de heroína, según consumo o no de cocaína en cinco ciudades de Colombia.

\section{Método}

Se encuestaron 1017 usuarios de drogas inyectables mayores de 18 años. Por medio de muestreo generado por el entrevistado, se calcularon razones de prevalencia crudas (OR) y ajustadas a través de una regresión logística, con sus respectivos IC $95 \%$ según el consumo de cocaína.

\section{Resultados}

El 60\% de los usuarios de heroína inyectada consumían también cocaína. Esta población en gran parte eran hombres, solteros, menores de 25 años, pertenecientes a estratos socioeconómicos bajos e involucrados en el microtráfico. Los comportamientos de riesgo en la última inyección de la droga fueron mayores en quienes también consumían cocaína. El uso de condón en la última relación sexual fue menor y se observó una mayor prevalencia de $\mathrm{VIH}$ en los consumidores de cocaína.

\section{Discusión y conclusión}

Se logró determinar que los usuarios de drogas inyectables que consumen cocaína se enfrentan a una alta probabilidad de presentar $\mathrm{VIH}$ y menor uso del condón, así como mayores comportamientos de riesgo sexual y relacionados con la inyección. Se requiere atención con medidas preventivas y sociales que disminuyan el policonsumo y las consecuencias de estas drogas en Colombia.

Palabras clave: Heroína, cocaína, VIH, conducta sexual, hepatitis C. 


\section{INTRODUCCIÓN}

Se estima que en América existen aproximadamente un millón y medio de personas que consumen heroína inyectada; ${ }^{1}$ en Colombia algunos registros indican que al menos 31852 personas han consumido heroína alguna vez en la vida. ${ }^{2} \mathrm{El}$ aumento en el uso de heroína inyectada se ha explicado por el efecto más intenso y duradero de esta droga y un mayor acceso, dada la disminución en los costos para adquirirla. ${ }^{3}$ Así, desde el inicio del consumo de heroína ha existido una creciente preocupación por el aumento de enfermedades de transmisión parenteral en las que se destaca el VIH y la hepatitis C. ${ }^{4}$

Al consumo de heroína inyectada se le agrega el uso concomitante de cocaína, la que en algunas partes del mundo es considerada la segunda droga de abuso más utilizada en los usuarios de heroína (entre el 15 - 60\%). El consumo de cocaína característicamente se produce por vía inhalatoria o intravenosa en los consumidores de heroína inyectada; ${ }^{5}$ cuando es usada por medio de esta última vía suele ocurrir el fenómeno llamado "speedball" que hace referencia a la inyección simultánea de cocaína y heroína con el fin de dar mayor rapidez y duración a los efectos, y se presenta aproximadamente en el 58\% de los usuarios de drogas inyectables (UDI). ${ }^{6,7}$

Se ha descrito que las personas que consumen cocaína presentan una mayor actividad sexual y un uso inconsistente del condón, lo que aumentaría el riesgo de presentar infecciones de transmisión sexual ${ }^{8,9}$ incluyendo el VIH. ${ }^{10}$

El consumo de estas dos drogas se convierte en un gran problema de salud pública, ya que a la par del aumento en el consumo de ellas, el número de personas que muere por sobredosis de heroína se ha incrementado de 0.7 a 2.7 muertes por cada 100 mil habitantes entre el 2002-2013 en Estados Unidos, ${ }^{11}$ y se ha encontrado que la mortalidad puede aumentar 63 veces con respecto a poblaciones sin dependencia a la droga. ${ }^{12}$ En cuanto al uso de la cocaína, su consumo está en cerca del $0.4 \%$ de la población mundial de entre 15-69 años. ${ }^{13}$ En países como Estados Unidos es la principal causa de consulta en urgencias por abuso en el consumo de sustancias psicoactivas $(40.3 \%$ ) y el $6.3 \%$ de visitas por suicido involucran a esta droga. ${ }^{14}$

En actividades ilegales como el microtráfico, se ha visto que aproximadamente el $16 \%$ de las personas que participan en ella son consumidores de más de una sustancia psicoactiva. ${ }^{15-17}$ En Colombia existen registros de personas que se inician en esta actividad para financiar sus propias adicciones y ser independientes económicamente. ${ }^{18}$

Por lo anterior, esta investigación tiene como fin identificar el consumo concomitante de cocaína en usuarios de heroína inyectable en Colombia y explorar las diferencias en los factores sociodemográficos, conductas sexuales y de inyección de drogas en consumidores de heroína inyectable según consumo o no de cocaína.

\section{MÉTODO}

Se realizó un estudio descriptivo transversal utilizando el muestreo de Respondent Driven-Sampling (RDS por sus siglas en inglés) $\cdot{ }^{19,20}$ La información se recolectó en el primer semestre del año 2014, teniendo como criterios de inclusión ser usuario activo de drogas inyectables y haber estado al menos seis meses inyectándose, haber cumplido entre 18 y 59 años de edad, disponer de un cupón de RDS vigente y firmar el consentimiento informado.

\section{Participantes}

El reclutamiento se inicia con un grupo de "semillas" o participantes no aleatorios de la población objetivo. En el caso de la presente investigación se determinó una cantidad inicial de tres semillas de las posibles para cada ciudad. El cálculo del tamaño de la muestra se realizó mediante la fórmula para una proporción, utilizando un nivel de precisión absoluta del $5 \%$ y un nivel de confianza $1-\alpha=95 \%$. El tamaño mínimo de la muestra por cada ciudad era de 196 personas. Estas ciudades fueron Bogotá, capital de Colombia, ubicada en el Departamento céntrico de Cundinamarca y ciudad con mayor población del país; Armenia, capital del Departamento de Quindío y ciudad con altas tasas reportadas de VIH y VHC en personas que se inyectan drogas (PQID); ${ }^{21}$ Cúcuta, capital del Departamento de Norte de Santander al noreste colombiano, ubicado en la frontera colombo-venezolana; Pereira, capital del Departamento de Risaralda, al occidente del país; y Medellín, segunda ciudad más poblada de Colombia, ubicada en el Departamento de Antioquia al noroccidente del país.

\section{Procedimientos}

Se utilizó un instrumento aplicado en el año 2010 con UDI de Medellín y Pereira, ${ }^{22}$ el cual tomó como base la encuesta de la Organización Mundial de la Salud (OMS), validada en la ciudad de Bogotá en el año 2000;23 este formulario incluía preguntas en torno a características demográficas, conductas de riesgo (divididas entre aquellas presentadas en los últimos seis meses y de la última inyección de heroína), la prueba de laboratorio en papel de filtro para detección de hepatitis $\mathrm{C} y$ $\mathrm{VIH},{ }^{24}$ con confirmación por prueba Western Blot para VIH en los casos requeridos. Los entrevistadores fueron capacitados previo a la recolección de la información como estrategia para controlar el sesgo de información así como para brindarles herramientas para asesorar a los participantes en caso de ser necesario.

\section{Análisis estadístico}

El análisis estadístico y los resultados fueron realizados en SPSS ${ }^{\circledR}$ 21.0. Para establecer la asociación entre las características de los usuarios de heroína inyectable según su 
Cuadro 1. Características sociodemográficas asociadas al consumo de cocaína en UDI

\begin{tabular}{|c|c|c|c|c|c|c|c|c|c|c|}
\hline \multirow[b]{3}{*}{ Variables } & \multicolumn{4}{|c|}{ Consumidores de cocaína } & \multirow[b]{3}{*}{$O R$} & \multirow[b]{3}{*}{$95 \%$ IC } & \multirow[b]{3}{*}{$p$} & \multirow[b]{3}{*}{$a O R$} & \multirow[b]{3}{*}{$95 \%$ IC } & \multirow[b]{3}{*}{$p$} \\
\hline & \multicolumn{2}{|c|}{ Sí } & \multicolumn{2}{|c|}{ No } & & & & & & \\
\hline & $n$ & $\%$ & $n$ & $\%$ & & & & & & \\
\hline \multicolumn{11}{|l|}{ Edad } \\
\hline$<25$ años & 327 & 53.8 & 198 & 48.4 & 1.24 & {$[0.96-1.59]$} & 0.09 & 1.26 & {$[0.98-1.63]$} & 0.07 \\
\hline$\geq 25$ años* & 281 & 46.2 & 211 & 51.6 & 1.00 & - & - & 1.00 & - & - \\
\hline \multicolumn{11}{|l|}{ Estrato } \\
\hline Bajo* & 391 & 64.5 & 299 & 73.1 & 1.00 & - & 0.01 & 1.00 & - & 0.03 \\
\hline Medio & 191 & 31.5 & 102 & 24.9 & 1.43 & {$[1.08-1.90]$} & 0.01 & 1.40 & {$[1.05-1.87]$} & 0.02 \\
\hline Alto & 24 & 4.0 & 8 & 2.0 & 2.29 & {$[1.02-5.18]$} & 0.05 & 2.00 & {$[0.87-4.59]$} & 0.10 \\
\hline \multicolumn{11}{|l|}{ Microtráfico } \\
\hline Sí & 365 & 61.1 & 199 & 49.4 & 1.61 & {$[0.63-1.34]$} & 0.00 & 1.57 & {$[1.21-2.03]$} & 0.00 \\
\hline No* & 232 & 38.9 & 204 & 50.6 & 1.00 & - & - & 1.00 & - & - \\
\hline
\end{tabular}

OR: Frecuencias anómalas.

IC: Intervalo de confianza.

aOR: Frecuencias anómalas ajustadas.

*Categoría de referencia.

consumo de cocaína, se utilizaron las pruebas estadísticas $\chi^{2}$ y Exacta de Fisher, con un nivel de significancia estadística del 5\%. Así mismo, se calcularon odds ratio crudos (OR) y ajustados a través de regresión logística, con sus respectivos IC 95\%. No se realizó imputación de los datos faltantes.

\section{Consideraciones éticas}

El proyecto fue avalado por el Comité de Ética de la Universidad CES.

\section{RESULTADOS}

\section{Características sociodemográficas}

De los 1017 usuarios de heroína inyectada, el 86.8\% (879) eran hombres y un 13.1\% (133) mujeres; el 50\% de los hombres tenía una edad de 26 años y las mujeres una edad mediana de 24 años. Con respecto a los consumidores de heroína inyectada y uso concomitante de cocaína, el $86.2 \%$ eran hombres, sin diferencias estadísticamente significativas con los usuarios que no consumían cocaína (cuadro 1).

Cuadro 2. Comportamientos de riesgo en las relaciones sexuales asociados al consumo de cocaína en UDI

\begin{tabular}{|c|c|c|c|c|c|c|c|c|c|c|}
\hline \multirow[b]{3}{*}{ Variables } & \multicolumn{4}{|c|}{ Consumidores de cocaína } & \multirow[b]{3}{*}{$O R$} & \multirow[b]{3}{*}{$95 \%$ IC } & \multirow[b]{3}{*}{$p$} & \multirow[b]{3}{*}{$a O R$} & \multirow[b]{3}{*}{$95 \%$ IC } & \multirow[b]{3}{*}{$p$} \\
\hline & \multicolumn{2}{|c|}{ Sí } & \multicolumn{2}{|c|}{ No } & & & & & & \\
\hline & $n$ & $\%$ & $n$ & $\%$ & & & & & & \\
\hline \multicolumn{11}{|c|}{ Utilización del condón en la última relación sexual } \\
\hline Sín & 259 & 43.0 & 207 & 51.47 & 1.00 & - & - & 1.00 & - & - \\
\hline No & 343 & 57.0 & 196 & 48.60 & 1.40 & {$[1.09-1.80]$} & 0.01 & 3.77 & {$[1.08-13.17]$} & 0.04 \\
\hline \multicolumn{11}{|c|}{ Utilización del condón en la última relación sexual con su pareja estable } \\
\hline Sí & 66 & 24.7 & 49 & 31.2 & 1.38 & {$[0.89-2.14]$} & 0.15 & 0.77 & {$[0.23-2.53]$} & 0.67 \\
\hline No* & 201 & 75.3 & 108 & 68.8 & 1.00 & - & - & 1.00 & - & - \\
\hline \multicolumn{11}{|c|}{ Utilización del condón en la última relación sexual con su pareja ocasional } \\
\hline Sí & 233 & 60.5 & 142 & 68.6 & 1.43 & {$[1.00-2.04]$} & 0.05 & 1.27 & {$[0.46-3.46]$} & 0.65 \\
\hline No* & 152 & 39.5 & 65 & 31.4 & 1.00 & - & - & 1.00 & - & - \\
\hline \multicolumn{11}{|l|}{$\mathrm{VIH}$} \\
\hline Sí & 38 & 6.3 & 16 & 3.9 & 1.64 & {$[0.90-2.98]$} & 0.11 & 1.88 & {$[1.02-3.45]$} & 0.04 \\
\hline No* & 570 & 93.8 & 393 & 96.1 & 1.00 & - & - & 1.00 & - & - \\
\hline \multicolumn{11}{|l|}{$\mathrm{VHC}$} \\
\hline Sí & 156 & 25.7 & 135 & 33.0 & 0.70 & {$[0.53-0.92]$} & 0.01 & 0.67 & {$[0.50-0.88]$} & 0.00 \\
\hline No* & 452 & 74.3 & 274 & 67.0 & 1.00 & - & - & 1.00 & - & - \\
\hline
\end{tabular}

OR: Frecuencias anómalas.

IC: Intervalo de confianza.

aOR: Frecuencias anómalas ajustadas.

*Categoría de referencia. 
Cuadro 3. Comportamientos de riesgo en la última inyección asociados al consumo de cocaína en UDI

\begin{tabular}{|c|c|c|c|c|c|c|c|c|c|c|}
\hline \multirow[b]{3}{*}{ Variables } & \multicolumn{4}{|c|}{ Consumidores de cocaína } & \multirow[b]{3}{*}{$O R$} & \multirow[b]{3}{*}{$95 \%$ IC } & \multirow[b]{3}{*}{$p$} & \multirow[b]{3}{*}{$a O R$} & \multirow[b]{3}{*}{$95 \%$ IC } & \multirow[b]{3}{*}{$p$} \\
\hline & \multicolumn{2}{|c|}{ Sí } & \multicolumn{2}{|c|}{ No } & & & & & & \\
\hline & $n$ & $\%$ & $n$ & $\%$ & & & & & & \\
\hline \multicolumn{11}{|c|}{ Compartió la mezcla de droga con alguien } \\
\hline Sí & 97 & 16.5 & 42 & 11.4 & 1.54 & {$[1.05-2.27]$} & 0.03 & 1.67 & {$[1.05-2.68]$} & 0.03 \\
\hline No* & 490 & 83.5 & 327 & 88.6 & 1.00 & - & - & 1.00 & - & - \\
\hline \multicolumn{11}{|c|}{ Estaba la droga en una cápsula o ampolleta lista para inyectarse } \\
\hline Sí & 25 & 4.3 & 6 & 1.6 & 2.69 & {$[1.09-6.62]$} & 0.03 & 3.44 & {$[1.21-9.78]$} & 0.02 \\
\hline No* & 562 & 95.7 & 363 & 98.4 & 1.00 & - & - & 1.00 & - & - \\
\hline \multicolumn{11}{|c|}{ Usted inyectó a otra persona } \\
\hline Sí & 34 & 5.8 & 10 & 2.7 & 2.21 & {$[1.08-4.52]$} & 0.03 & 2.10 & {$[0.94-4.68]$} & 0.07 \\
\hline No* & 553 & 94.2 & 359 & 97.3 & 1.00 & - & - & 1.00 & - & - \\
\hline
\end{tabular}

Entre la población estudiada se encontró que el 51.6\% (525) de los encuestados eran menores de 25 años. Al comparar a las personas según el consumo de cocaína, se observó una mayor proporción de este uso en menores de 25 años en relación con los mayores de esta edad ( $53.8 \%$ vs. $46.2 \%)$. El 79\% de los consumidores de heroína identificados eran solteros.
El estrato socioeconómico de la familia era bajo en el $68 \%$ (690) de los usuarios, medio en el $28.9 \%$ (293) y alto en el 3.2\% (32). Se encontró una mayor proporción en el consumo de heroína inyectada y cocaína en el estrato socioeconómico alto comparado con el estrato bajo y medio (OR: 2.29 IC 95\% 1.02-5.18).

El 56.4\% (564) de la población encuestada se vio asociada con el microtráfico; el 61\% eran UDI y consumidores

Cuadro 4. Comportamientos de riesgo en los últimos seis meses asociados al consumo de cocaína en UDI

\begin{tabular}{|c|c|c|c|c|c|c|c|c|c|c|}
\hline \multirow[b]{3}{*}{ Variables } & \multicolumn{4}{|c|}{ Consumidores de cocaína } & \multirow[b]{3}{*}{$O R$} & \multirow[b]{3}{*}{$95 \%$ IC } & \multirow[b]{3}{*}{$p$} & \multirow[b]{3}{*}{$a O R$} & \multirow[b]{3}{*}{$95 \%$ IC } & \multirow[b]{3}{*}{$p$} \\
\hline & \multicolumn{2}{|c|}{ Sí } & \multicolumn{2}{|c|}{ No } & & & & & & \\
\hline & $n$ & $\%$ & $n$ & $\%$ & & & & & & \\
\hline \multicolumn{11}{|c|}{ Se ha inyectado con jeringas que tuvieran la mezcla de droga lista o premezclada por alguien conocido } \\
\hline Sí & 142 & 21.0 & 64 & 14.7 & 1.54 & {$[1.11-2.13]$} & 0.01 & 1.29 & {$[0.90-1.85]$} & 0.16 \\
\hline No & 533 & 79.0 & 371 & 85.3 & - & - & - & - & - & - \\
\hline \multicolumn{11}{|c|}{ Ha divido con otra persona la mezcla pasándola de jeringa } \\
\hline Sí & 179 & 27.1 & 97 & 23.2 & 1.23 & {$[0.92-1.64]$} & 0.15 & 0.92 & {$[0.65-1.29]$} & 0.64 \\
\hline No & 481 & 72.9 & 322 & 76.8 & - & - & - & - & - & - \\
\hline \multicolumn{11}{|c|}{ Ha compartido con otras personas algodones, agua de enjuague o cucharas cuando estaba inyectando } \\
\hline Sí & 348 & 51.8 & 182 & 57.7 & 1.46 & {$[1.14-1.86]$} & 0.00 & 1.20 & {$[0.89-1.63]$} & 0.22 \\
\hline No & 324 & 48.2 & 248 & 42.3 & - & - & - & - & - & - \\
\hline \multicolumn{11}{|c|}{ Ha sacado dosis de una mezcla de droga que otras personas también estaban compartiendo } \\
\hline Sí & 279 & 41.6 & 124 & 28.5 & 1.78 & {$[1.37-2.31]$} & 0.00 & 1.57 & {$[1.13-2.16]$} & 0.00 \\
\hline No & 392 & 58.4 & 311 & 71.5 & - & - & - & - & - & - \\
\hline \multicolumn{11}{|c|}{ Se ha inyectado alguna vez en un recinto cerrado al que la gente va a consumir, como una "olla" o un sitio de inyección } \\
\hline Sí & 416 & 61.7 & 292 & 67.3 & 0.78 & {$[0.60-1.01]$} & 0.06 & 0.74 & {$[0.56-0.97]$} & 0.31 \\
\hline No & 258 & 38.3 & 142 & 32.7 & - & - & - & - & - & - \\
\hline \multicolumn{11}{|c|}{ Alguna vez lo ha inyectado una persona que cobra por inyectar } \\
\hline Sí & 70 & 10.4 & 66 & 15.2 & 0.64 & {$[0.45-0.92]$} & 0.02 & 0.54 & {$[0.36-0.79]$} & 0.00 \\
\hline No & 601 & 89.6 & 367 & 84.8 & - & - & - & - & - & - \\
\hline \multicolumn{11}{|c|}{ Ha inyectado alguna vez con una jeringa casera o hechiza } \\
\hline Sí & 43 & 6.4 & 15 & 3.5 & 1.89 & {$[1.04-3.46]$} & 0.36 & 1.95 & {$[1.02-3.74]$} & 0.43 \\
\hline No & 631 & 93.6 & 418 & 96.5 & - & - & - & - & - & - \\
\hline
\end{tabular}

OR: Frecuencias anómalas.

IC: Intervalo de confianza.

aOR: Frecuencias anómalas ajustadas.

*Categoría de referencia. 
de cocaína, mientras que los que no consumían cocaína reportaron una menor proporción en actividades relacionadas con el microtráfico (49.4\%) (cuadro 1); siendo 1.6 veces el riesgo en los consumidores de cocaína de hacer parte de actividades de microtráfico (OR: 1.61 IC 95\% 0.63-1.34) (cuadro 2).

\section{Comportamientos sexuales}

Se encontró que el 57\% de los UDI no usó el condón en la última relación sexual, comparado con un $48.6 \%$ de UDIs que no consumían cocaína y tampoco usaron condón (OR: 1.4 IC 95\% 1.09-1.8); de igual manera se observó que la última relación sexual con pareja estable en los dos grupos se asoció con un menor uso del condón.

El 5.4\% de los UDI fue identificado con VIH; de los UDI no consumidores de cocaína, el 3.9\% tenía VIH, comparado con el $6.3 \%$ de los UDI que consumía cocaína (OR: 1.64 IC 95\% 0.9-2.9). No se observó relación en los UDI que consumían cocaína para el aumento de riesgo de VHC (OR: 0.7 IC 95\% 0.53-0.92).

\section{Conductas de riesgo en la inyección}

El $16.5 \%$ de los UDI que consumían cocaína compartían la mezcla de droga, mientras que un $11.4 \%$ de los UDI que no consumían cocaína también la compartían. Así mismo, fue más frecuente el consumo de drogas en cápsulas o ampolletas listas para inyectarse, en UDI que consumían cocaína (4.3\% vs. $1.6 \%$ ) (cuadro 3 ).

Al preguntarles si habían inyectado a otra persona, se encontró que el 5.8\% de las personas que consumían cocaína y eran UDI lo hacían, comparado con el $2.7 \%$ de los UDI no consumidores de cocaína.

Al realizar el análisis con el fin de obtener medidas ajustadas, se observó que al comparar los UDI que consumen cocaína frente a los que no consumen, el ser menor de 25 años aumenta el riesgo 1.26 veces de consumir cocaína. Pertenecer a un estrato socioeconómico alto aumenta el riesgo dos veces más de consumir cocaína. Así mismo, se encontró una asociación del microtráfico 1.57 veces en quienes consumían cocaína frente los que no la consumían.

Se identificó que las personas que consumían cocaína presentaban casi cuatro veces el riesgo de tener relaciones sexuales sin usar condón y dos veces de presentar VIH (cuadro 4). En las conductas de riesgo en la última inyección, sobresale que compartir la mezcla de droga es 1.67 veces mayor en los que consumen cocaína que en los que no lo hacen. A la vez consumir la droga en cápsulas listas para inyectarse se presenta 3.4 veces en los UDI que consumen cocaína frente a los que no la usan. Se observó además que el consumo de cocaína en UDI aumentaba tres veces el riesgo de inyectar a otra persona (cuadro 4).

\section{DISCUSIÓN Y CONCLUSIÓN}

Esta investigación buscaba identificar el consumo concomitante de cocaína en usuarios de heroína inyectable en Colombia y explorar las diferencias según el consumo o no de cocaína en las conductas de riesgo y características sociodemográficas de los UDI. En el mundo se ha visto un aumento considerable del consumo de drogas psicoactivas que generan alta dependencia como la cocaína y la heroína, ${ }^{1}$ esta última de uso más reciente en la población colombiana ${ }^{25}$ convirtiéndose en un problema de salud pública, que toma cada vez más fuerza en algunas ciudades de Colombia. A esta problemática se le agrega la alta prevalencia del policonsumo en personas que inician el uso de heroína inyectada. ${ }^{26}$

Los UDI y consumidores de cocaína se han descrito como poblaciones con un bajo nivel de escolaridad, altos niveles de pobreza, asociados al microtráfico y menores de 30 años de edad. ${ }^{1,7,27-30}$ En esta investigación se identificó una alta prevalencia del consumo de cocaína y heroína inyectada en personas menores de 25 años, comportamiento que incluye a los adolescentes y adultos jóvenes como un grupo de riesgo para el consumo de drogas. ${ }^{31}$

En relación a lo descrito en otras investigaciones sobre la frecuencia de consumo de drogas como la cocaína y heroína en población de estrato bajo, ${ }^{1,29}$ en este estudio se observó un aumento importante del consumo de cocaína en personas de estrato alto que podría asociarse con una mayor facilidad económica para adquirir la cocaína y las drogas de uso inyectable, a la vez que estos sectores han sido reconocidos por tener un menor control en la distribución de drogas ilícitas. ${ }^{32}$

Floyd et al. observaron una mayor actividad de microtráfico en personas que consumían drogas ilícitas y otros estudios han reforzado esta asociación en los policonsumidores. ${ }^{16,17}$ En el presente estudio se logró identificar una mayor relación de los consumidores de heroína y cocaína en esta actividad, probablemente por la mayor accesibilidad en las redes de microtráfico al obtener varias drogas de abuso para su consumo. ${ }^{17,33}$

La literatura médica ha mostrado que el consumo concomitante de heroína y cocaína es más frecuente en la población masculina que en la femenina, similar a la proporción de género vista en el consumo de otras drogas psicoactivas. ${ }^{5,34}$ En este estudio no se encontraron diferencias a lo descrito y se observó que el consumo de cocaína en consumidores de heroína se presentó de manera más usual en hombres.

Los UDI reportan una mayor prevalencia de VIH explicado por conductas poco higiénicas en el uso compartido de jeringas y un mayor comercio sexual para obtener dinero; ${ }^{35,36}$ así mismo, se ha observado una asociación en el uso de la cocaína con una mayor actividad sexual y un uso inconsistente del condón. ${ }^{8,9}$ En el presente estudio se encontró que el uso de cocaína en UDI se asociaba con una menor utilización del condón y mayor prevalencia de VIH que los UDI no consumidores de cocaína. 
En la última inyección, el uso de cocaína en UDI se asoció con un mayor riesgo de compartir la mezcla de droga con alguien y de inyectar a otra persona, señalando que estas medidas poco higiénicas en el momento de la aplicación de la droga, se relacionan con un aumento del riesgo de transmisión parenteral de infecciones. Similar a lo encontrado en otros estudios, donde se ha relacionado la alta presencia de $\mathrm{VIH}$, hepatitis $\mathrm{C}$ y otras enfermedades en UDI y policonsumidores con mayores conductas de riesgo en el momento de la inyección. ${ }^{3,4,37}$

\section{Limitaciones}

Al ser una investigación transversal, no se logró establecer la secuencia temporal sin sustentar inferencias de causalidad. En algunos casos, los individuos pueden olvidar detalles pertinentes en algunas preguntas a responder, al estar basados en el auto reporte. Por último, el RDS es considerado una muestra dependiente de la red social, la cual puede tener limitaciones al reportar las estimaciones poblacionales en los usuarios de drogas inyectables por ciudad, afectando la generalización de los resultados obtenidos.

En este estudio se identificó un mayor consumo de cocaína en UDI menores de 25 años, de estratos socioeconómicos altos, involucrados en redes de microtráfico, los cuales deben ser atendidos con políticas sociales que generen una disminución de la comercialización y el uso de estas sustancias de manera insegura.

\section{Financiamiento}

Esta investigación se realizó en el marco del convenio de asociación 00317 con el Ministerio de Justicia del Derecho y la Universidad CES, en el que se buscaba identificar la prevalencia de VIH y hepatitis $\mathrm{C}$ y los comportamientos de riesgo en usuarios de drogas inyectables.

\section{Conflicto de interes}

Los autores declaran no tener ningún conflicto de intereses.

\section{REFERENCIAS}

1. Organizacion Panamericana de la Salud. Epidemiología del uso de drogas en America Latina y el Caribe: Un enfoque de salud pública; 2009.

2. Ministerio de la Protección Social, Dirección Nacional de Estupefacientes. Estudio nacional sobre consumo de sustancias psicoactivas en Colombia. [Internet]. 2013 [cited 2015 Sep 25]. Available from: https:// www.unodc.org/documents/colombia/2014/Julio/Estudio_de_Consumo_UNODC.pdf

3. Mejía IE, Gómez AP. La inyección de drogas en Bogotá: una amenaza creciente [Internet]. Adicciones. 2005 [citado septiembre 25, 2015].

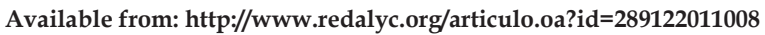

4. Bassani S, Toro C, De la Fuente L, Teresa Bruga M et al. Prevalencia de infección por virus de transmisión parenteral en consumidores actuales de heroína de 3 ciudades españolas. Med Clínica 2004;122(15):570572 .
5. Bobes J, Sáiz PA, Bascarán MT, González Rodríguez MP. Epidemiología del uso/abuso de cocaína. Adicciones 2001;13(Supl 2):23-36.

6. Leri F, Stewart J, Tremblay A, Bruneau J. Heroin and cocaine co-use in a group of injection drug users in Montréal. J Psychiatry Neurosci JPN 2004;29(1):40-47.

7. Broz D, Wejnert C, Pham HT, DiNenno E et al. HIV infection and risk, prevention, and testing behaviors among injecting drug users -- National HIV Behavioral Surveillance System, 20 U.S. cities, 2009. Morb Mortal Wkly Rep Surveill Summ Wash DC 2002. 2014;63(6):1-51.

8. Raj A, Saitz R, Cheng DM, Winter M et al. Associations between alcohol, heroin, and cocaine use and high risk sexual behaviors among detoxification patients. Am J Drug Alcohol Abuse 2007;33(1):169-178.

9. Borders TF, Stewart KE, Wright PB, Leukefeld $C$ et al. Risky sex in rural America: longitudinal changes in a community-based cohort of methamphetamine and cocaine users. Am J Addict Am Acad Psychiatr Alcohol Addict 2013;22(6):535-542.

10. Marta Torrens LS. Cocaine abuse among heroin addicts in Spain. Drug Alcohol Depend 1991;27(1):29-34.

11. Jones CM, Logan J, Gladden RM, Bohm MK. Vital Signs: demographic and substance use trends among heroin users - United States, 2002-2013. MMWR Morb Mortal Wkly Rep 2015;64(26):719-725.

12. Grönbladh L, Ohlund LS, Gunne LM. Mortality in heroin addiction: impact of methadone treatment. Acta Psychiatr Scand 1990;82(3):223227.

13. United Nations Office on Drugs and Crime. World Drug Report 2015 [Internet]. Vienna, Austria; [citado septiembre 26, 2015]. Report No.: 15. Available from: https://www.unodc.org/documents/wdr2015/ World_Drug_Report_2015.pdf

14. Substance Abuse and Mental Health Services Administration. Drug abuse warning network, 2011: National estimates of drug-related emergency department visits. Rockville: Report No.: 13-4760.

15. Vaughn MG, Salas-Wright CP, DeLisi M, Shook JJ et al. A typology of drug selling among young adults in the United States. Subst Use Misuse 2015;50(3):403-413.

16. Kirst MJ, Erickson P, Strike C. Poly-substance use among male and female street youth in Toronto, Canada. Int J Soc Inq 2009;2:123-130.

17. Floyd LJ, Alexandre PK, Hedden SL, Lawson AL et al. Adolescent drug dealing and race/ethnicity: a population-based study of the differential impact of substance use on involvement in drug trade. Am J Drug Alcohol Abuse 2010;36(2):87-91.

18. Pérez Gómez A. Microtráfico de heroina, un tercer estudio realizado en Medellín y Bogota en el 2009. Colombia: Ministerio de Proteccion Social; 2010.

19. Respondent Driven Sampling [Internet]. [citado julio 8, 2014]. Available from: http://www.respondentdrivensampling.org/

20. McCreesh N, Copas A, Seeley J, Johnston LG et al. Respondent driven sampling: determinants of recruitment and a method to improve point estimation. Plos One 2013;8(10):e78402-e78402.

21. Berbesi-Fernández D, Segura-Cardona Á, Montoya-Vélez L, Castaño-Perez GA. Hepatitis C y VIH en usuarios de drogas inyectables en Armenia-Colombia. Adicciones 2015;27(4):246-252.

22. Berbesi D, Montoya L, Segura A. Prevalencia de VIH y comportamientos de riesgo en UDI en Medellin y Pereira. Bogota: Ministerio de Proteccion Social; 2012.

23. Mejía IE, Gómez AP. La inyección de drogas en Bogotá: una amenaza creciente. Adicciones 2005;17(3):251-260.

24. Pérez Guevara MT, Rolo Gómez FM, Nibot Sánchez C, Cruz Sui O et al. Determinación de anticuerpos al virus de la inmunodeficiencia humana tipo 1 (VIH-1) en muestras de sangre seca en papel de filtro. Rev Cubana Med Trop 1998;50(2):93-95.

25. Gómez AP. Transiciones en el consumo de drogas en Colombia [Internet]. Adicciones. 2009 [citado septiembre 25, 2015]. Available from: http://www.redalyc.org/articulo.oa?id=289122882010

26. Leri F, Bruneau J, Stewart J. Understanding polydrug use: review of heroin and cocaine co-use. Addiction 2003;98(1):7-22. 
27. Higgs P, Owada K, Hellard M, Power R et al. Gender, culture and harm: an exploratory study of female heroin users of Vietnamese ethnicity. Cult Health Sex 2008;10(7):681-696.

28. Wagner FA, González C, Aguilera RM, Ramos LE et al. Oportunidades de exposición al uso de drogas entre estudiantes de secundaria de la Ciudad de México [Internet]. Salud Mental 2003 [citado septiembre 25, 2015]. Available from: http://www.redalyc.org/articulo.oa?id=58222603

29. Bernstein E, Bernstein J, Tassiopoulos K, Valentine A et al. Racial and ethnic diversity among a heroin and cocaine using population: Treatment system utilization. J Addict Dis 2005;24(4):43.

30. Hunt $D$. Stealing and dealing: cocaine and property crimes. NIDA Res Monogr 1991;110:139-150.

31. Ortiz A. Tendencias y uso de cocaína en adolescentes y jóvenes de la Ciudad de México. Sistema de reporte de información en drogas. Salud Ment 2005;28(2):91-97.

32. Torres ICS, Quintero MA. El consumo de alcohol, tabaco y otras drogas, como parte del estilo de vida de jóvenes universitarios. Rev Colomb Psicol 2004;13(1):74-89.
33. Moyle L, Coomber R. Earning a score: An exploration of the nature and roles of heroin and crack cocaine "user-dealers". Br J Criminol 2015;55(3)534-555.

34. EMCDDA. 2015 Annual report on the state of the drugs problem in Europe. Lisboa, Portugal: 2015.

35. Berbesi D, Segura-Cardona A, Montoya-Vélez L, Mateu-Gelabert P. Consumo de heroína inyectada en Colombia y comportamientos de riesgo [Internet]. Salud Mental 2013 [citado septiembre 25, 2015]. Available from: http://www.redalyc.org/articulo.oa?id=58225671004

36. Lum PJ, Sears C, Guydish J. Injection risk behavior among women syringe exchangers in San Francisco. Subst Use Misuse 2005;40(11):1681-1696.

37. Mwatelah RS, Lwembe RM, Osman S, Ogutu BR et al. Co-infection burden of hepatitis $C$ virus and human immunodeficiency virus among injecting heroin users at the Kenyan Coast. PloS One 2015;10(7):e0132287. 